\title{
EFFECT OF GRID-ADAPTIVE INTERPOLATION OVER DEPTH IMAGES
}

\author{
Arbaaz Singh \\ Department of Computer science \& Engineering, \\ Indian Institute of Technology, Ropar, Punjab, India \\ arbaazseitrpr.ac.in
}

\begin{abstract}
A suitable interpolation method is essential to keep the noise level minimum along with the timedelay. In recent years, many different interpolation filters have been developed for instance H.264-6 tap filter, and AVS- 4 tap filter. This work demonstrates the effects of a four-tap lowpass tap filter (Grid-adaptive filter) on a hole-filled depth image. This paper provides (i) a general form of uniform interpolations for both integer and sub-pixel locations in terms of the sampling interval and filter length, and (ii) compares the effect of different finite impulse response filters on a depth-image. Furthermore, the author proposed and investigated an integrated Grid-adaptive filter, that implement hole-filling and interpolation concurrently, causes reduction in time-delay noticeably along with high PSNR.
\end{abstract}

\section{KEYWORDS}

Depth Images, Hole filling, Interpolation, Interpolation filter

\section{INTRODUCTION}

In 3-D computer graphics, a depth map is an image or image channel that contains information relating to the distance of the surfaces of scene objects from a viewpoint [1]. Once the original image and depth image is given, 3-D can be synthesized by mapping pixel coordinates one by one according to its depth value. It is the next emerging revolution after the high definition video and is the key technology in advanced three dimensional television systems (3-D TV) and free-view television systems [2-4]. A new member of 3-D sensor family, kinect has drawn great attention of researchers in the field of 3-D computer vision for its advantage of consumer price and real time nature. Based on a structured light technique, Kinect is able to generate depth and colour images at a speed of about $30 \mathrm{fps}$ [5]. However, limited by depth measuring principle and object surface properties, the depth image captured by the Kinect contains missing data as well as noise. These areas of missing data are known as holes. Holes appear due to sharp horizontal changes in depth image, thus the location and size of holes differ from frame to frame. Several attempts are made to remove the noise and filling of holes with the correct data to make it suitable for different applications by means of bilateral and median-filters [6]. Apart from hole-filling, image interpolation as well occurs in all digital pictures at several stages [7]. Interpolation is the process of determining the values of a function at positions lying between its samples. It achieves this process by fitting a continuous function through the discrete input samples. This permits input values to be evaluated at arbitrary positions excluding those defined at the sample points. Interpolation is required to produce a larger image than the one captured and finds an imperative

Natarajan Meghanathan et al. (Eds) : WiMONe, NCS, SPM, CSEIT - 2014

pp. 185-189, 2014. (C) CS \& IT-CSCP 2014

DOI : $10.5121 / \mathrm{csit} .2014 .41214$ 
consign in transmission of 3-D images. 3-D images have been used in robotic guidance, product profiling and object tracking, in battle preparation, medical diagnosis and many more [8]. These all applications require both hole-filling and interpolation to provide a preferred output. A suitable interpolation method is essential to keep the noise level minimum along with the time-delay. The standard/conventional procedure to interpolate a depth image is to first fill the holes and then apply the interpolation filter that leads to low PSNR and a great time-complexity. In recent years, many different interpolation filters have been developed for instance H.264-6 Tap filter, AVS- 4 tap filter and so on [9-10].

The standard/conventional procedure to interpolate a depth image is to first fill the holes and then apply the interpolation filter that leads to low PSNR and a great time-complexity. Recently, a texture-adaptive hole-filling algorithm is proposed for post-processing of rendered image on 3D video to save the computational cost [11]. The algorithm first determines the type of holes, and then fills the missed pixels in raster-order depending upon hole types and texture gradient of neighbors with simple data operation, which benefits for fast processing. Further, the quality of virtual view images from rendering is demonstrated by establishing a connection of pixel coordinate warping between reference image and virtual image in the rendering process, to make a quick decision on the position of hole region and its edge [12]. Subsequently, by outward expanding the edge of holes, warping error pixels are covered. Then, hole-filling is through using use mean filter and the image restoration method after eliminating the false contour by image synthesis. Nonlinear filters called Spline Adaptive Filters (SAFs), implementing the linear part of the Wiener architecture with an IIR filter instead of an FIR one are also come into picture to improve the PSNR and computational delay [13]. This paper investigates a four-tap Grid-adaptive filter on a hole-filled depth image that provides uniform interpolations for both integer and subpixel locations in terms of the sampling interval and filter length. Further, it compares with other different finite impulse response filters and investigates the integrated grid adaptive filter to reduce the time delay.

\section{THEORETICAL \& EXPERIMENTAL ANALYSIS}

The depth image $I$ is a 2D grid of $K_{V} x K_{H}$ pixels. The pixels denote the distance of the objects in real scene according to its image co-ordinates [4]. The holes are areas in the image that have invalid (missing) data. Each pixel has 8 neighbouring pixels that share a face or a vertex with the centre pixel. Our goal is to go through the image and fill up the holes in the image. Let each pixel in the image be denoted as $I(v, h)$, where $v, h$ can be any integer value between 0 and $K_{V}, K_{H}$ respectively. If $I(v, h)=0$, then that pixel is a part of a hole. A $3 \times 3$ gaussian weighted averaging filter is used to fill the holes. The filter takes the weighted average of the depth values of its neighbours and replaces the hole with the obtained averaged value. The weights are decided such that the neighbour pixels, that are holes themselves, are ignored while the non-zero valued pixels are used to find the new value of the hole-pixel.

Let the vertical and horizontal distances between the two nearest known pixels be $T_{v}$ and $T_{h}$, respectively. Subsequently, the aim is to insert and interpolate $N_{v}$ and $N_{h}$ pixels within the intervals of $T_{v}$ and $T_{h}$ respectively. So, after the interpolation, we will have a sum of $\left[K_{v}\left(1+N_{v}\right)-N_{v}\right] \times\left[K_{h}\left(1+N_{h}\right)-N_{h}\right]$ pixels and the sampling intervals for vertical and horizontal directions will be changed to $D_{v}=T_{v} /\left(1+N_{v}\right)$ and $D_{h}=T_{h} /\left(1+N_{h}\right)$ respectively. For example, Figure 1 shows the case of $N_{v}=N_{h}=1$ with $D_{v}=T_{v} / 2$ and $D_{h}=T_{h} / 2$. 


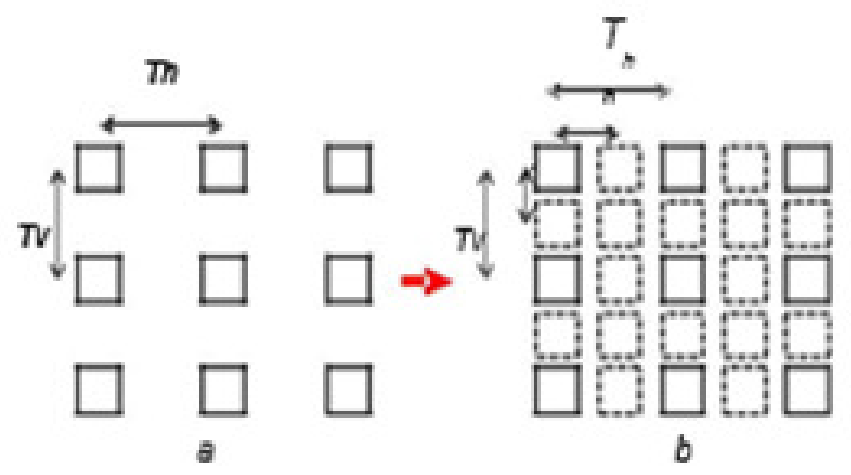

Figure 1. Uniformly spaced interpolation (a) before interpolation with known (bold square) pixels, (b) After Interpolation for missing (dotted square) pixels

Therefore, the general formula for 1-D interpolation filter will be

$$
y(K T+n \Delta)=\sum_{m=M+1}^{M} y(K T+m T) P(m T-n \Delta)
$$

Where, $n$ denotes the pixel location to be interpolated within two reference samples i.e. $(K T)$ and $(K T+T)$. So, we need to evaluate equation (1) for all $(1-N)$. The expression (1) covers both fractional and integral pixel interpolations. For the fractional locations, the value of $\Delta$ is kept less than 1 while for integral locations the value of $\Delta$ is always greater than 1 . The proposed-4 tap filter is obtained using the Langrage interpolation, which is used to generalise the linear interpolation by approximating the sinc function [10]. The Lagrange interpolation kernel is an $L^{\text {th }}$ order polynomial function determined by $L+l$ values in the following function,

$$
P(t+m T-n \Delta)=\frac{Q_{m T-n \Delta}(t)}{Q_{m T-n \Delta}(m T-n \Delta)}
$$

Where,

$$
Q_{m T-n \Delta}(t)=\prod_{k}^{(t-(k t-n \Delta))} /(t-(m t-n \Delta))
$$

For any sampling grid layout and scale, the filter coefficients can be calculated by fitting (3) to the grid. The PSNR is measured for different filters such as the linear averaging filter, H.264-6 Tap filter with coefficients $(1,-5,20,20,-5,1) / 32$, the AVS- 4 Tap filter with coefficients $(-1$, $5,5,-1) / 8$ and the proposed-4 tap grid adaptive filter with coefficients $(-1,9,9,-1) / 16$ to recommend the best one. All test images are taken from the Middlebury Database (2006) and are expanded as the same sampling layout and scale of the proposed 4-Tap filter i.e. doubling the number of rows and columns with $T=2, N=1, M=2$, and $\Delta=1$ ). For evaluating the performance the simulated filters, all images are expanded at the same sampling layout and scale of the filters (i.e. doubling the number of rows and columns with $T=2, N=1, M=2$, and $D=1$ ). For computation, the images are reduced to half before interpolation so that the size of image remains same after hole-filling and interpolation. The PSNR is calculated between a perfect image and its noisy approximation and can be easily defined via mean squared error 
$(M S E)$. For a given noise-free $m \times n$ monochrome image ' $I$ ' and its noisy approximation ' $K$ ', $M S E$ is defined as:

$M S E=\frac{1}{m n} \sum_{i=0}^{m-1} \sum_{j=0}^{n-1}\left(I(i, j)-K(i, j)^{2}\right.$

and $P S N R$ is measured as

$P S N R=10 \log _{10}\left(\frac{I^{2}{ }_{M A X}}{M S E}\right)=P S N R=20 \log _{10}\left(\frac{I_{M A X}}{M S E}\right)$

As shown in the Table 1, although the filter length is shorter than H.264-6, the proposed 4-Tap filter yields the highest average PSNR. The obtained results show that the proposed-4 filter with the coefficients $(-1,9,9,-1) / 16$ has almost equal influence to the H.264 filter in terms of PSNR evaluation. Consequently, it is suggested to recommend H.264-6 filter to produce high quality images. Otherwise, the proposed-4 filter is a better option that provides high quality images along with minimal time processing delays as it is used four level tapping. Further, besides the PSNR values, the time-complexity of the proposed integrated adaptive filter is also evaluated. Conventionally, RGBD images are enhanced by implementing interpolation and hole-filling algorithm independently but it leads to a large amount of time-complexity along with low PSNR . In this work, the author proposed an integrated adaptive filter that implements hole-filling and interpolation concurrently. The time-complexity of the conventional filter is deliberated as $O\left(9 * n^{2}\right)$ though the proposed integrated filter has a time-complexity of $O\left(8 * n^{2}\right)$. Accordingly, it is obvious that a reduction of $O\left(n^{2}\right)$ is reported through the projected filtering scheme.

Table 1. Measured PSNR using different Filters

\begin{tabular}{|c|c|c|c|c|}
\hline \multirow{2}{*}{ Image } & \multicolumn{4}{|c|}{ Measured PSNR } \\
\cline { 2 - 5 } & $\begin{array}{c}\text { Linear } \\
\text { Average } \\
\text { Filter }\end{array}$ & $\begin{array}{c}\text { AVS- 4 Tap } \\
\text { Filter }\end{array}$ & $\begin{array}{c}\text { H.264- 6 Tap } \\
\text { Filter }\end{array}$ & $\begin{array}{c}\text { Grid } \\
\text { Adaptive-4 } \\
\text { Tap Filter }\end{array}$ \\
\hline Aloe & 39.9108 & 40.0318 & 40.0684 & 40.0846 \\
\hline Baby 2 & 47.109 & 47.2672 & 47.2149 & 47.2845 \\
\hline Baby 3 & 47.1809 & 47.4044 & 47.2218 & 47.4055 \\
\hline Bowling 2 & 44.2 & 44.4352 & 44.3577 & 44.4295 \\
\hline Cloth & 47.6444 & 47.7766 & 47.7397 & 47.8162 \\
\hline Cloth 3 & 49.6144 & 49.7569 & 49.6258 & 49.7836 \\
\hline Computer & 42.7804 & 43.0139 & 42.0716 & 42.9928 \\
\hline Flowerpots & 45.0702 & 45.2199 & 45.0892 & 45.2617 \\
\hline Lampshade 2 & 44.0775 & 44.3312 & 44.1833 & 44.3026 \\
\hline Rocks & 51.6279 & 51.8097 & 51.6726 & 51.8243 \\
\hline
\end{tabular}

\section{CONCLUSIONS}

To demonstrate the power of grid adaptive filter, the PSNR is measured for different filters to determine the best one. In our experiments, it has been shown that the grid adaptive four-tap filter yields the highest average PSNR values (almost same as that of the six-tap filter). Moreover, it offers minimum time delays than that of six-tap filter on integrating hole-filling and interpolation 
in tandem. Accordingly, it is suggested to use the proposed integrated Grid adaptive filter for the enhancement of depth images.

\section{ACKNOWLEDGEMENT}

I thank to Professor Chee Sun Won, Department of Electronics and Electrical Engineering, Dongguk University, Seoul, South Korea for his kind and valuable guidance for carrying out this work. I also thank to the Department of Electronics and Electrical Engineering, Dongguk University, Seoul, South Korea for providing lab facility and technical support.

\section{REFERENCES}

[1] Depth Map, Wikipedia accessed on 17th July, 2014, http://en.wikipedia.org/wiki/Depth_map

[2] Kubota, A. Smolic, M. Magnor, M. Tanimoto, T. Chen, and C. Zhang, (2007) "Multi-view Imaging and 3DTV”, IEEE Signal Processing Magazine, Vol. 24, No. 6, pp 10-21.

[3] T. Fujii, and M. Tanimoto, (2002) "Free viewpoint TV system based on ray-space representation", in proceeding of Three Dimensional TV, Video and Display, 175, SPIE 4864, 1st November, 2002, doi:10.1117/12.454905

[4] S. L. Forman, and L. A. Steen, "Case study: Image Processing", http://www.stolaf.edu/people/steen/Projects/ ATE/imp.html.

[5] Li Chen, Hui Lin, and Shutao Li, (2012) "Depth image enhancement for Kinect using region growing and bilateral filter", 21st International Conference on Pattern Recognition (ICPR 2012), Tsukuba, Japan, pp 3070 - 3073.

[6] S. Matyunin, D. Vatolin, Y. Berdnikov, and M. Smirnov, (2011) "Temporal filtering for depth maps generated by Kinect depth camera", 3DTV Conference: The True Vision-Capture, Transmission and Display of 3D Video (3DTV-CON), pp 1-4, Doi: 10.1109/3DTV.2011.5877202

[7] Digital Image Interpolation, http://www.cambridgeincolour.com/tutorials/ image-interpolation.htm

[8] Andrew Wilson, "3D imaging systems target multiple applications", http://www.visionsystems.com/articles/print/volume-18/issue-9/features/3d-imaging-systems-target-multipleapplications.html

[9] Lehmann, T.M., Gonner, C., and Spitzer, K., (1999) 'Survey: interpolation methods in medical image processing', IEEE Trans. Med. Imag., Vol. 18, No. 11, 1999, pp 1049-1075.

[10] Chee Sun Won, (2013) "Grid Adaptive Interpolation Filter", Electronic Letters, Vol. 49, No. 3, pp 181-182, Doi: 10.1049/el.2012.2481

[11] Linwei Zhu, Mei Yu, Gangyi Jiang, Xiangying Mao, Songyin Fu, Ting Luo, “A New Virtual View Rendering Method based on Depth Map for 3DTV3DTV; virtual view rendering; depth; false contour; holes filling; image restoration”, Procedia Engineering, Volume15, pp.1115-1119, 2011

[12] Michele Scarpiniti, Danilo Comminiello, Raffaele Parisi, Aurelio Uncini,“ Nonlinear system identification using IIR Spline Adaptive Filters”, Signal Processing, Volume 108, pp.30-35, March 2015

[13] Chee Sun Won, "Grid Adaptive Interpolation Filter”, Electronic Letters, Volume 49, Issue 3, pp. 181182, 31st January 2013, doi: 10.1049/el.2012.2481

\section{AUTHOR}

I, Arbaaz Singh am currently studying in the field of Image Processing in the department of Computer Science \& Engineering at Indian Institute of Technology, Ropar, Punjab, India. I worked as a research Intern at Dongguk University under the kind guidance of Professor Chee Sun Won in the field of Depth Images. My areas of interest are Depth image processing and designing issues of Interpolation filters.

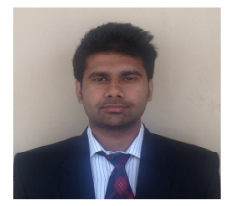

